\title{
In-Vitro screening on the interaction of Chaetomium Cellulolyticum Chachal \& D. Hawksworth with Aspergillus flavus link Ex Fr. and Curvularia lunata (Wakker) Boedijin
}

\begin{abstract}
This study generally aimed to determine the interaction of Chaetomium cellulolyticum with Aspergillus flavus and Curvularia lunata and the antifungal properties of Chaetomium cellulolyticum extracts against of Aspergillus flavus and Curvularia lunata. Description of the fungal microscopic association was done in slide bi-culture while the zone of colonization was studied in the co-culture method and zone of inhibition was determined in the antimicrobial bioassay. Results revealed that in slide bi-culture test, the hyphae of A. flavus were damaged when grown together with C. cellulolyticum. Co-culture method revealed that $C$. cellulolyticum does not colonize the agar lawn of A. flavus. In bioassay, results revealed that at $24^{\text {th }}$ to $48^{\text {th }}$ hours of incubation, mycelial crude hexane and ethanol extracts do not produced zone of inhibition on A. flavus. Statistical analysis showed that the zone of inhibition of the two crude extracts were comparable to the positive and negative control. However, at $72^{\text {nd }}$ to $96^{\text {th }}$ hours, the extracts were significantly smaller to the positive control and comparable to the negative control $(\mathrm{p}<0.05)$.

In slide bi-culture test revealed that the hyphae and conidia of $C$. lunata were broken when grown together with $C$. cellulolyticum. In Co-culture method results revealed that $C$. cellulolyticum colonized the agar lawn of $C$. lunata by $0.15 \%$. In Bioassay, results revealed that mycelial crude hexane and ethanol extracts do not produced zones of inhibition on C. lunata at $24^{\text {th }}$ to $96^{\text {th }}$ hours of incubation. Analysis of variance and comparison among means showed, that inhibitions of two extracts were comparable to the positive and negative control at $24^{\text {th }}$ hours of incubation. However, at $48^{\text {th }}$ to $96^{\text {th }}$ hours of incubation, the crude extracts were significantly smaller to the positive control but comparable to the negative control $(\mathrm{p}<0.05)$. Chaetomium cellulolyticum have antibiosis mechanism to deform or damage the morphological hyphae and either conidia of A. flavus and C. lunata. However, the affectivity of crude hexane and ethanol extracts from C. cellulolyticum were not observed to inhibit the growth of A. flavus and C. lunata.
\end{abstract}

Keywords: plant pathogens, fungicides, antagonistic activity
Volume 2 Issue I - 2015

\author{
Mark Anthony Santiago Cadelina, Cynthia C \\ Divina \\ Department of Biological Sciences, Central Luzon State \\ University, Philippines
}

Correspondence: Mark Anthony Santiago Cadelina, Department of Biological Sciences, Central Luzon State University, Science City of Munoz, Nueva Ecija, Philippines, Email cadelinamark@gmail.com

Received: October 27, 2014 | Published: March 02, 2015
Abbreviations: LSD, laboratory service division; CPC, crop protection cluster; $\mathrm{PDB}$, potato dextrose broth; PDA, potato dextrose agar; DMSO, dimethyl sulfoxide; CRD, completely randomized design; ANOVA, analysis of variance; DMRT, Duncan's multiple range test; SPSS, statistical package for social sciences

\section{Introduction}

\section{Background of the study}

The search for natural fungicides has been dominating the research arena in the past years. The Environmental Protection Agency ${ }^{1}$ described fungicides as pesticides applied to kill or inhibit growth of fungi that cause economic damage. Copper compounds, especially copper sulfate mixed with lime and water and sulfur and synthetic organic compounds have long been used for this purpose. EPA ${ }^{1}$ added that plants, fungi and microorganisms contain antifungal substances that occur naturally that they could be tapped as possible source. The ability of many fungi to antagonize various microorganisms and other fungi has been well established. In most cases, attention has been centered upon the antagonistic actions of various fungi against organisms capable of causing plant diseases. EPA ${ }^{1}$ listed fungi in the groups of Ophiobolus, Rhizoatonia, and Fusarium. Aspergillus and Chaetomium capable of antagonizing pathogenic organisms. The search for promising microbial antagonists have been of increasing interest and used to control plant diseases. ${ }^{2}$

Seeds are regarded as highly effective means for transporting plant pathogens over long distances. Seed-borne diseases have been found to affect the growth and productivity of crop plants. ${ }^{3} \mathrm{~A}$ seed-borne pathogen present externally or internally or associated with seed as contaminant, may cause seed abortion, seed rot, seed necrosis, reduction or elimination of germination capacity as well as seedling damage resulting in development of disease at later stages of plant growth by systemic or local infection. ${ }^{4}$ Moisture and seed nutrient content in the midrange of seed development provide optimum conditions for fungal development and toxin production. Mycotoxins are carcinogenic metabolites produced by fungus such as aflatoxins liberated by pathogenic fungus Aspergillus flavus Link ex Fr. and Phytotoxins produced by Curvularia lunata Boedijin ${ }^{5}$ as contaminations in maize (Zea mays L.) grain. Aflatoxin contamination of maize kernels poses a serious health hazard to both humans and 
animals. ${ }^{6}$. A. flavus infects kernels via the corn silk or in association with insect damage. They are colored yellow green and as the spores age they turn a dark green. ${ }^{7}$ C. lunata often formed on the edge of the leaf and become brown, oval to irregular. It is also reported to cause head blight in foreign countries. ${ }^{8}$ Chaetomium is a large ascomycetous fungus producing perithecia. It is found on a variety of substrates containing cellulose including paper and plant compost. It can be readily found on the damaged paper in sheetrock. Chaetomium spp. have a worldwide distribution. They have biological control in plant diseases especially vegetable and fruits. They protect plants from diseases by producing many kinds of antibiotics and ergosterols that can suppress plant pathogens, stimulate growth of plants and induce resistance of plants. ${ }^{9}$ Biological products are useful, not only for the protection against plant diseases, but can also be used for curative effects of plant diseases, and also promote plant growth. ${ }^{10}$

\section{Significance of the study}

Many bioactive compounds from different fungi have been reported to inhibit many plant pathogenic fungi. Results of this study created an impact and significant contribution in biotechnology, particularly in the field of agriculture to limit the damage of plant pathogens in both conventional and organic farming. The information achieved was provided full support to develop new generation of biofungicide that will substitute to the hazardous chemical fungicides against seed-borne fungal pathogens, and promoted plant disease biocontrol in practice not only in the Philippines but also in the world.

\section{Scope and limitation of the study}

Strains of seed-borne fungal pathogens (Aspergillus flavus and Curvularia lunata) and Chaetomium cellulolyticum that were used in this study were obtained at the UP-Natural Sciences Research Institute Culture Collection (UPCC) of University of the Philippines, Diliman, Quezon City; Laboratory Service Division (LSD) of Philmec, Science City of Munoz, Nueva Ecija and the Department of Plant Pathology, Crop Protection Cluster (CPC) of College of Agriculture, University of the Philippines, Los Banos, Laguna. This study was focus only on the interactions between C. cellulolyticum and the pathogens, and on antagonistic effect of Chaetomium cellulolyticum against seed-borne fungal pathogens.

\section{Time and place of the study}

This study was conducted from December 2010 to March 2011 at the Department of Biological Sciences, College of Arts and Sciences, Central Luzon State University, Science City of Munoz, Nueva Ecija, and at PhD Biotechnology in Plant Pathology Laboratory, Biocontrol Research Unit and Mycology Section, Department of Plant Pest Management, Faculty of Agricultural Technology, King Mongkut's Institute of Technology Ladkrabang, Bangkok, Thailand.

\section{Objectives of the study}

This study generally aimed to determine the interaction of Chaetomium cellulolyticum with Aspergillus flavus and Curvularia lunata and the antifungal properties of Chaetomium cellulolyticum extracts against of Aspergillus flavus and Curvularia lunata. It specifically aimed to:

i. Describe the interaction between C. cellulolyticum and A. flavus.

ii. Determine the zone of colonization of C. cellulolyticum on agar lawn of A. flavus. iii. Determine the zone of inhibition of mycelial crude extracts of C. cellulolyticum on A. flavus.

iv. Describe interaction between C. cellulolyticum and C. lunata.

v. Determine the zone of colonization of C. cellulolyticum on agar lawn of C. lunata and

vi. Determine the zone of inhibition of mycelial crude extracts of C. cellulolyticum on C. lunata.

\section{Review of related literature}

\section{Chaetomium spp.}

Chaetomium is a large fungal genus species of which are found worldwide on variety of substrates including damp sheetrock, carpet, plant compost, soil and other substrates containing cellulose. It is also commonly found on deteriorating wood products. Chaetomium is an ascomycete that produces ascospores in dense, hairy fruiting bodies called perithecia. Perithecia are flask-shaped structures with a hole through which spores are extruded. The perithecial hairs can take a variety of forms depending upon the species. The ascospores are dark brown and often lemon-shaped. They collect in a dense mass just outside the perithecium. The perithecia, surrounded by the long hairs, can be as large as $1 \mathrm{~mm}$, and are visible to the naked eye. Surfaces supporting Chaetomium growth with sporulation may superficially resemble old colonies of Stachybotrys. In culture, colonies of Chaetomium are rapidly growing, cottony and initially white in color. As the colonies mature, they become gray to olive in color and from the reverse the color is tan to red or brown to black. Chaetomium species grow best at between 25 and $35^{\circ} \mathrm{C}$ [11]. Charoenporn et al. [10] used Potato Dextrose Broth (PDB) media for mycelial matting of Chaetomium sp. The optimum $\mathrm{pH}$ of PDB for Chaetomium is at 4.5 to $5.5 \mathrm{pH}$. Chaetomium cellulolyticum is a thermo tolerant fungus rather than thermophilic, optimal growth resulted at $37^{\circ} \mathrm{C}$ but temperatures above $40^{\circ} \mathrm{C}$ were inhibitory.

\section{Materials and methods}

\section{Test organisms}

Preparation of samples: The strain of Chaetomium cellulolyticum and seed-borne fungal pathogens (Aspergillus flavus and Curvularia lunata) were obtained from the UP-Natural Sciences Research Institute Culture Collection (UPCC) of University of the Philippines, Diliman, Quezon City; Laboratory Service Division (LSD) of Philmec, Science City of Munoz, Nueva Ecija and the Department of Plant Pathology, Crop Protection Cluster (CPC) of College of Agriculture, University of the Philippines, Los Banos, Laguna. Sterilized Potato Dextrose Agar (PDA) was used as medium for the mycelial propagation of the samples. The pure cultures of the organisms were used as source and sub culturing was done by touching the spores using sterile inoculating needle. Triple point inoculation, where in the distances of the three (3) point growth are equal, was used in inoculating the medium. These sub-cultures were incubated at room temperature.

\section{Interaction studies}

The slide bi-culture test and co-culture method were done to determine interactions of the C. cellulolyticum with plant pathogens.

Slide bi-culture: Ten milliliter $(10 \mathrm{ml})$ of sterilized PDA was poured in the Petri dish. After it had cooled, this was sliced into blocks like the size of the cover slip using sterile scalpel and this was aseptically 
inoculated using the L- shape inoculating needle in the sterile glass slide. The spores at the edge of radial mycelial growth of the $C$. cellulolyticum was touched by sterile inoculating needle and used to inoculate the agar block and the sterile cover slip was placed. Same procedure was done using the pathogen. The distance between the antagonist and the pathogen was approximately one centimeter (1 $\mathrm{cm})$. The slide was placed in a sterile Petri dish and was lined with sterile paper as moist chamber. This was replicated three (3) times and incubated at room temperature. This was periodically observed everyday under compound microscope. The interaction of the antagonist and the pathogen was observed under photomicroscope at $\mathrm{PhD}$ Biotechnology in Plant Pathology Laboratory, Biocontrol Research Unit and Mycology Section, Department of Plant Pest Management, Faculty of Agricultural Technology, King Mongkut's Institute of Technology Ladkrabang, Bangkok, Thailand.

Co-culture method: Co-culture method was conducted to determine if $C$. cellulolyticum could colonize the agar lawn of the plant pathogens using the method of Soytong. ${ }^{12}$ Potato Dextrose Agar (PDA) at pH 7 was poured in the Petri plate and marked into two sections. Aseptically, approximately six millimeters $(6 \mathrm{~mm})$ of the seven (7) day old culture of C. cellulolyticum was bored using a sterile cork borer and this was inoculated at the half section of the agar along the middle line of the agar. The disc was tapped lightly to make the disc attach to the surface. The same method was applied using the pathogen. The Petri plates were marked properly and these were incubated in inverted position at room temperature. During incubation, the fungus that first crossed the midline was recorded. The zone of colonization of Chaetomium cellulolyticum was measured in millimeters $(\mathrm{mm})$ using vernier caliper and this was done by measuring along the middle mark. To determine the area of colonization, the width and the length of the mycelial growth was measured in every twenty four hours (24 hrs).

\section{Extract bio assay}

Experimental treatments: The study also looked into the inhibitory activities of crude extracts of $C$. cellulolyticum against the plant pathogens, A. flavus and C. lunata. There were four (4) treatments used in this study and each treatment was replicated five (5) times. These treatments were:

i. T1- Hexane extract of C. cellulolyticum

ii. T2- Ethanol extract of C. cellulolyticum

iii. T3- Positive Control (Dithane M-45)

iv. T4- Negative Control (Dimethyl sulfoxide)

\section{Preparation of crude extract}

Preparation of mycelial mat: One hundred milliliter $(100 \mathrm{ml})$ Potato Dextrose Broth (PDB) at $\mathrm{pH} 5.5$ was used as medium for propagation of Chaetomium cellulolyticum. The broth was sterilized in autoclave at one hundred twenty one degree Celsius $\left(121^{\circ} \mathrm{C}\right)$ for thirty minutes (30 mins). The seven (7) day old culture $C$. cellulolyticum was sliced into blocks with sterilized scalpel and this was aseptically inoculated in the prepared broth using sterile inoculating needle and incubated at room temperature for 30 days.

Ethanol and hexane extraction: After thirty (30) days, the fungal mycelia were removed from broth using sterile forceps and were placed in Petri dish to dry over night at room temperature. The extraction of C. cellulolyticum was performed by the method describe by Kanokmedhakul et al. ${ }^{13}$ Ten grams $(10 \mathrm{~g})$ dried mycelial mat was submerged and extracted in one hundred milliliter $(100 \mathrm{ml})$ $95 \%$ hexane and $95 \%$ ethanol for twenty four hours (24 hr) and was incubated at room temperature. And after extracted this was filtered using filter paper (Whatman no. 1). The solvents were separately evaporated in rotary evaporator under reduced pressure at fifty degree Celsius $\left(50^{\circ} \mathrm{C}\right)$ to yield crude hexane and ethanol respectively and this was done at Rice Chem Laboratory, Phil Rice, Maligaya, Science City of Munoz, Nueva Ecija. Each extract was dissolved in 2\% Dimethyl sulfoxide (DMSO).

\section{Paper disc bioassay}

Lawn preparation: Suspension of A. flavus and C. lunata were prepared by touching the spores using sterile inoculating needle and mixing in the ten milliliter $(10 \mathrm{ml})$ distilled water. Dilution was done and spore concentration of $1 \times 10^{6}$ spores $/ \mathrm{ml}$ of each fungus was determined using sterile heamacytometer and it was mixed with a prepared sterile PDA media. The paper discs were submerged in Ethanol extract, Hexane extract, Dithane M-45 and DMSO for twenty four (24) hours. Each lawn of the pathogens, two (2) discs was placed according to treatments. Each treatment was replicated five (5) times. The treatments were marked properly and this was incubated at room temperature for four (4) days.

Measuring of the zone of inhibition: During incubation, the inhibition zones from the center of the discs to the inner margin of the surroundings of fungal growth was measured in millimeters using vernier caliper in every twenty four hours for four (4) days and this was recorded.

\section{Data gathered}

The data gathered were:

i. The interaction between C. cellulolyticum and A. flavus.

ii. The zone of colonization of C. cellulolyticum on agar lawn of A. flavus.

iii. The zone of inhibition of mycelial crude extracts of $C$. cellulolyticum on A. flavus.

iv. Interaction between C. cellulolyticum and C. lunata.

v. The zone of colonization of C. cellulolyticum on agar lawn of C. lunata and

vi. The zone of inhibition of mycelial crude extracts of $C$. cellulolyticum on C. lunata.

\section{Experimental layout}

The experiment was laid out following Completely Randomized Design (CRD). The fugal interactions were qualitatively described and documented. Zones of inhibition were analyzed using Analysis of Variance (ANOVA) and compared using Duncan's Multiple Range Test (DMRT) at 5\% level of significance. Statistical Package for Social Sciences (SPSS) was used to analyze the data.

\section{Results and discussion}

\section{Interactions of Chaetomium cellulolyticum and Aspergillus flavus}

The interactions of $C$. cellulolyticum and A. flavus were studied using the slide bi-culture, co-culture techniques and extract bioassay. Description of the fungal microscopic association was done in slide 
bi-culture while the zone of colonization was studied in the co-culture method and zone of inhibition was determined in the antimicrobial bioassay.

Slide bi-culture: Slide bi-culture was done to determine the interactions between $C$. cellulolyticum and A. flavus under photomicroscope. Figure 1 shows some features of Chaetomium cellulolyticum and Aspergillus flavus photo microscopy. It shows the spores of $C$. cellulolyticum, normal hypha of A. flavus, damaged hyphae of $A$. flavus. It was observed that the hyphae of $A$. flavus were damaged when grown together with $C$. cellulolyticum. Based on the results, $C$. cellulolyticum may produce substances that could damage the hyphae of A. flavus. Similar observation was reported by Pornsuriya $^{14}$ about the reaction of some strains of Chaetomium like $C$. cochloides against some pathogens. It was reported that the pathogen showed abnormal features of hyphae and demonstrated uncommon shapes.

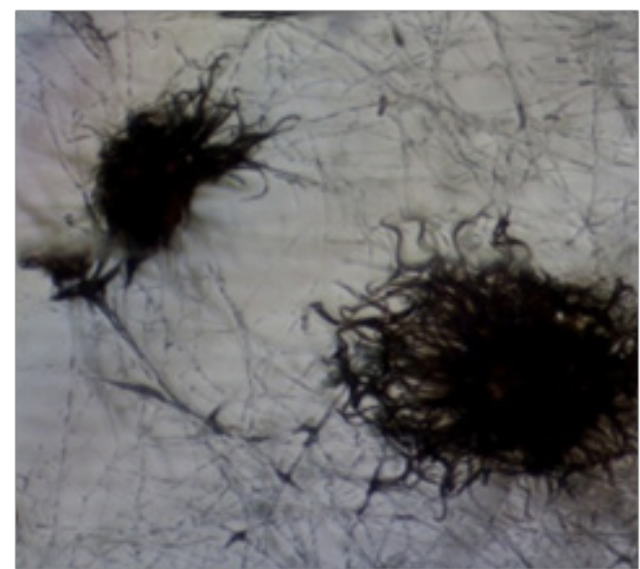

C. cellulolyticum Spores

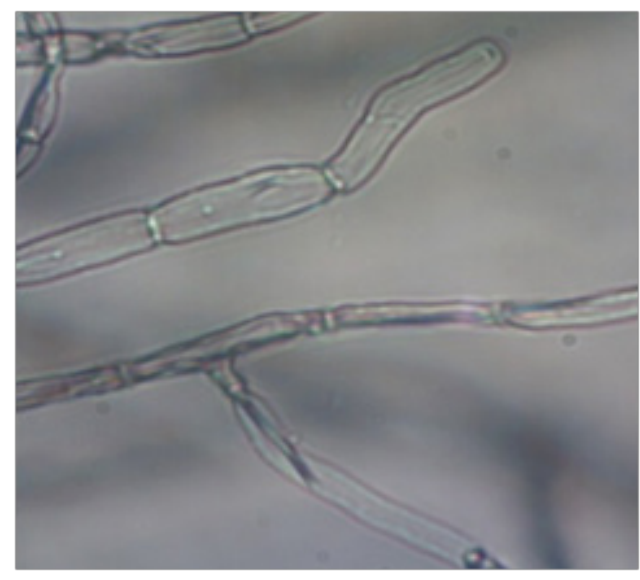

normal hyphae of A. flavus

Figure Ia Features of $C$. cellulolyticum and A. flavus under photomicroscope (HPO).
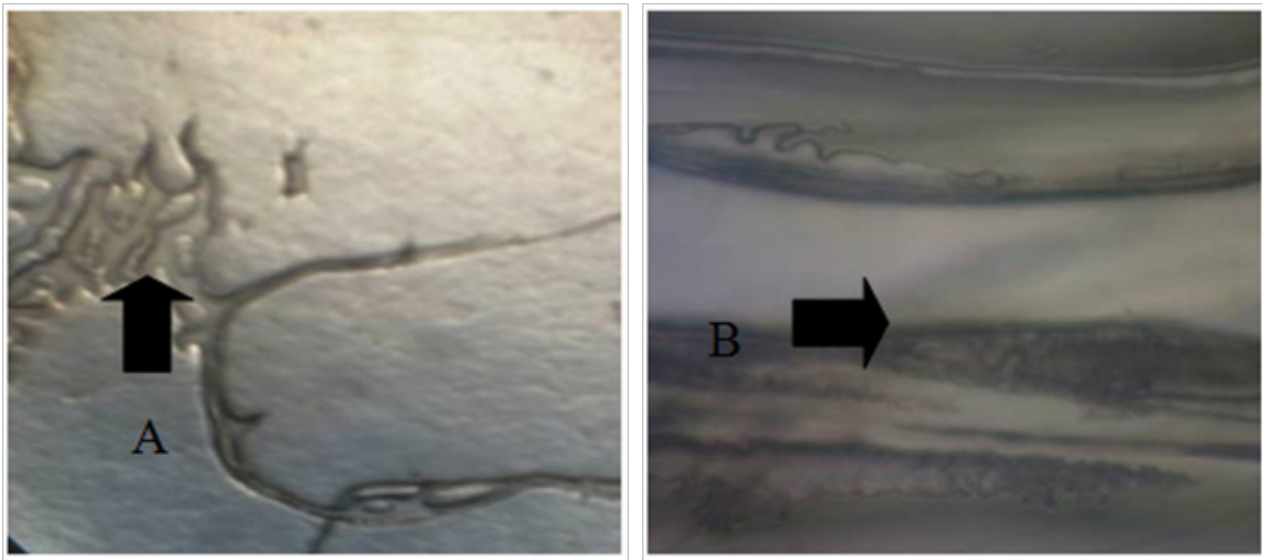

Damaged hyphae A. Flavus

Figure Ib Damaged hyphae of A. flavus (A and B) under photomicroscope (HPO).

Co-culture method: The C. cellulolyticum was tested to determine if this fungus could colonize the agar lawn of $A$. flavus using co-culture method as shown in Figure 2. The area and the percentage colonization of $C$. cellulolyticum and the pathogen were recorded at 30 days as shown in Figure 3. The results indicated that A. flavus ramified faster than $C$. cellulolyticum as shown in Figure 3. A. flavus colonized by $4,234.33 \mathrm{~mm}^{2}$ or $52.28 \%$ the agar lawn of C. cellulolyticum at 30 days and first crossed the midline. It is clearly observed that there was no colonization of C. cellulolyticum on agar lawn of A. flavus. On the other hand, the C. cellulolyticum colonized by $2,566 \mathrm{~mm}^{2}$ or $31.68 \%$. Results showed that, C. cellulolyticum was grown slowly, similarly as what Soytong ${ }^{15}$ said that Chaetomium spp. is a slow growing fungus compared to pathogens, and the growth and development is at 25 to 30 days.

Antimicrobial bioassay: The mycelia of $C$. cellulolyticum were extracted using Hexane and Ethanol extractions. This method was done to determine the presence of inhibitory effect of $C$. cellulolyticum extracts on the mycelial growth of A. flavus. Table 1 shows the mean diameter of zone of inhibition of all the treatments. Results revealed that at $24^{\text {th }}$ to $48^{\text {th }}$ hours of incubation see Figures 4 $\& 5$, mycelial crude hexane and ethanol extracts do not produced zone of inhibition on A. flavus. Statistical analysis showed that the zone of inhibition of the two crude extracts were comparable to the positive and negative control. However, at $72^{\text {nd }}$ to $96^{\text {th }}$ hours, the extracts were 
significantly smaller to the positive control and comparable to the negative control see Appendix I \& J. The affectivity of the mycelial crude extracts was not observed at $24^{\text {th }}$ to $96^{\text {th }}$ hours may because of the little amount of bioactive compounds found in the crude extracts.
The active components capability may also have short time effect to control the pathogen or the crude extracts were diffused across time in contrast to the report of Soytong et al. ${ }^{15}$ that substances extracted from Chaetomium spp. could exhibit activity towards some pathogens.
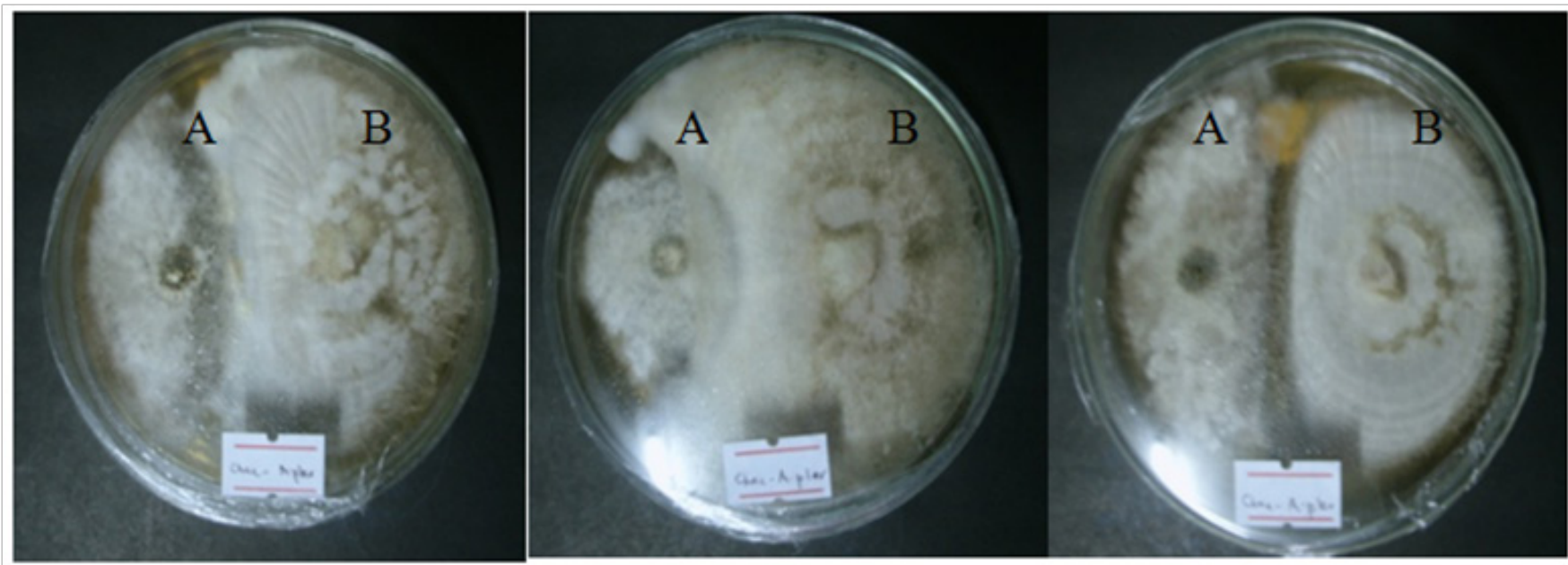

Figure 2 The co-culture of $C$. cellulolyticum (A) and A. flavus (B) per replicates at 30 days.

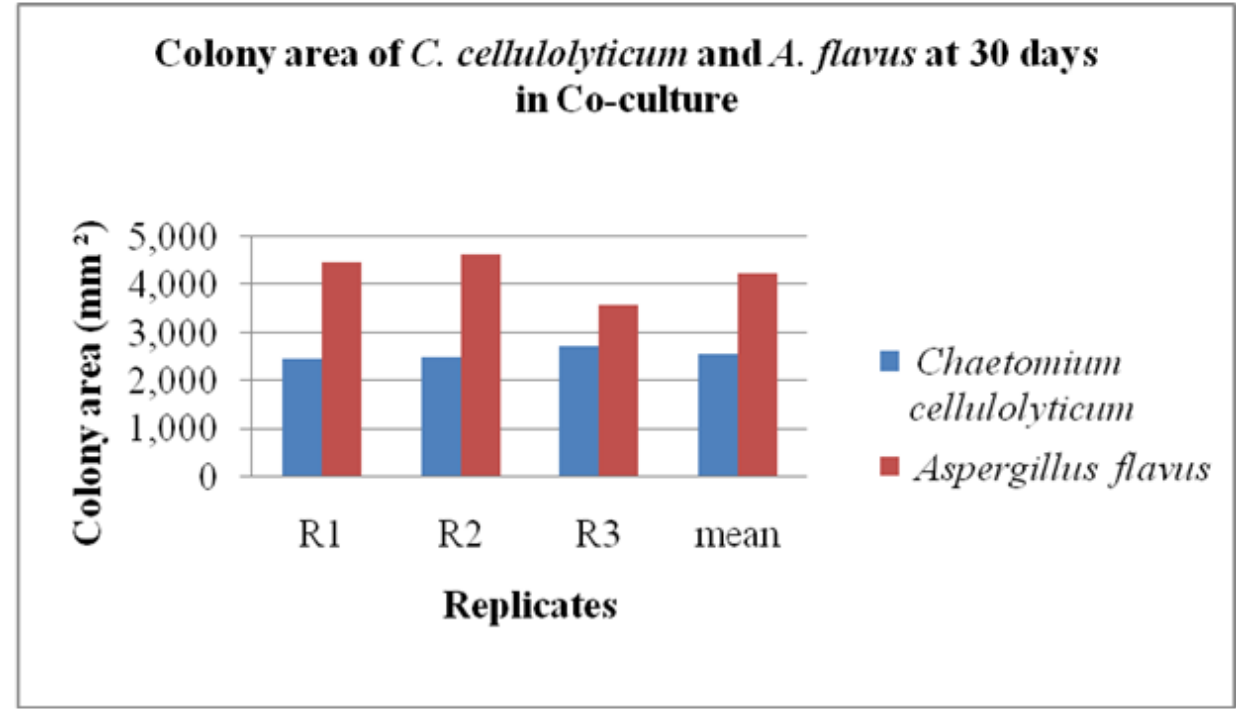

Figure 3 The colony area of $C$. cellulolyticum and A. flavus per replicates at 30 days in co-culture.

Table I Diameter of zone of inhibition of all the treatments against Aspergillus flavus

\begin{tabular}{lllll}
\hline Mean Diameter of Zone of Inhibition ( $\mathbf{m m})$ & & & \\
\hline Treatments & $\mathbf{2 4}^{\text {th }}$ Hour & $\mathbf{4 8}^{\text {th }}$ Hour & $\mathbf{7 2}^{\text {nd }}$ Hour & $\mathbf{9 6}^{\text {th }}$ Hour \\
\hline TI-Crude Hexane Extract & $6.00^{\mathrm{a}}$ & $6.00^{\mathrm{a}}$ & $6.00^{\mathrm{b}}$ & $6.00^{\mathrm{b}}$ \\
T2-Crude Ethanol Extract & $6.00^{\mathrm{a}}$ & $6.00^{\mathrm{a}}$ & $6.00^{\mathrm{b}}$ & $6.00^{\mathrm{b}}$ \\
T3-Dithane (Positive control) & $6.00^{\mathrm{a}}$ & $6.00^{\mathrm{a}}$ & $34.60^{\mathrm{a}}$ & $30.60^{\mathrm{a}}$ \\
T4-DMSO (Negative control) & $6.00^{\mathrm{a}}$ & $6.00^{\mathrm{a}}$ & $6.00^{\mathrm{b}}$ & $6.00^{\mathrm{b}}$ \\
\hline
\end{tabular}

Means followed by a common letter in each column are not significantly different by DMRT at $\mathrm{P}<0.05$. 


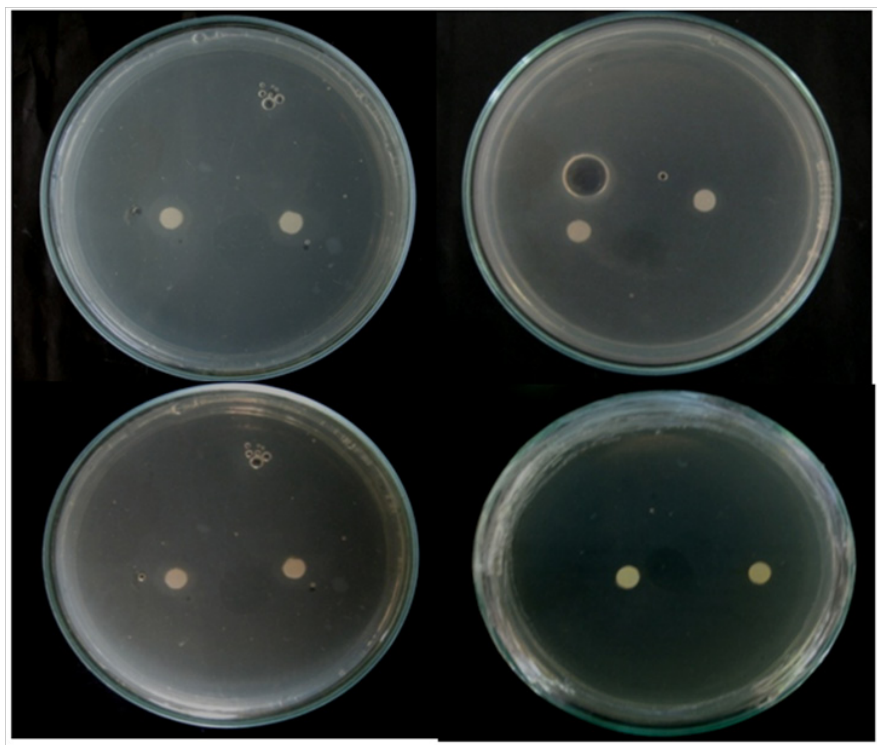

Figure 4 Zone of Inhibition exhibited by all the treatments against $A$. flavus at $24^{\text {th }}$ hours of incubation. (TI- crude hexane extract, T2- crude ethanol extract, T3- positive control (Dithane), T4- negative control (DMSO).
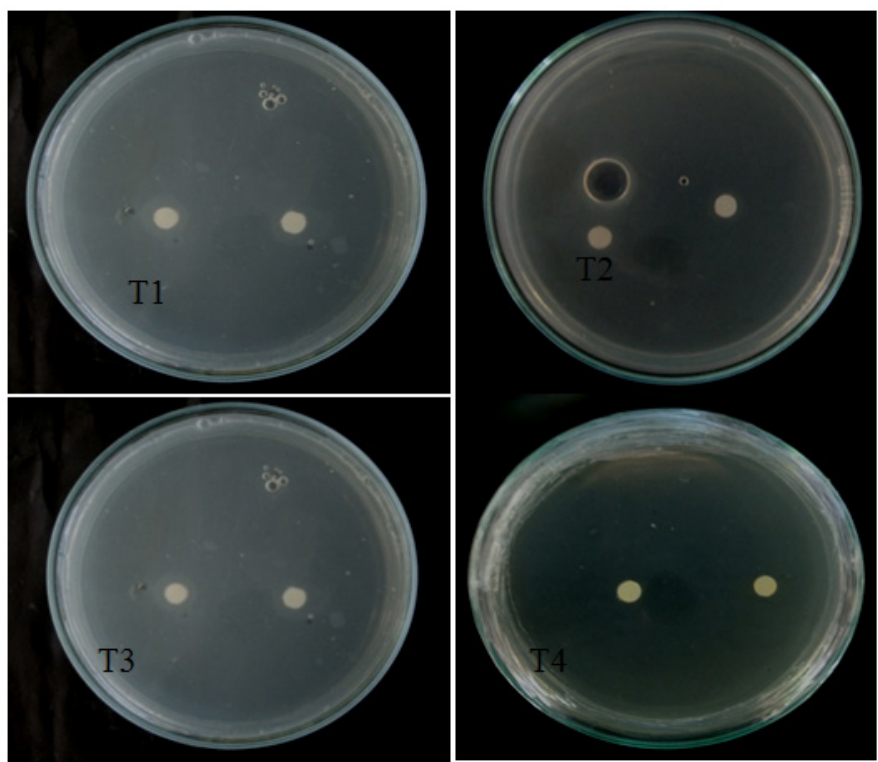

Figure 5 Zone of Inhibition exhibited by all the treatments against A. flavus at $48^{\text {th }}$ hours of incubation. (TI- crude hexane extract, T2- crude ethanol extract, T3- positive control (Dithane), T4- negative control (DMSO).

\section{Interactions of Chaetomium cellulolyticum and Curvularia lunata}

The interactions of C. cellulolyticum and C. lunata were also studied using the slide bi-culture, co-culture techniques and antimicrobial bioassay. Description of the fungal microscopic association was done in slide bi-culture while the zone of colonization was studied in the co-culture method and zone of inhibition was determined in the antifungal bioassay.

Slide bi-culture: This slide bi-culture was done to determine the interactions between $C$. cellulolyticum and $C$. lunata under photomicroscope. Figure 6 shows some features of $C$. lunata under photomicroscope. It shows the normal hyphae and conidia of $C$. lunata and damaged hyphae and conidia of $C$. lunata. Results revealed that the hyphae and conidia of $C$. lunata were broken when grown together with C. cellulolyticum. Based on the results, C. cellulolyticum may produce inhibitory substances that could break the hyphae and conidia of $C$. lunata. It was reported by Pornsuriya ${ }^{14}$ that the pathogen showed abnormal features of hyphae and demonstrated uncommon shapes when grown together with some strains of Chaetomium.
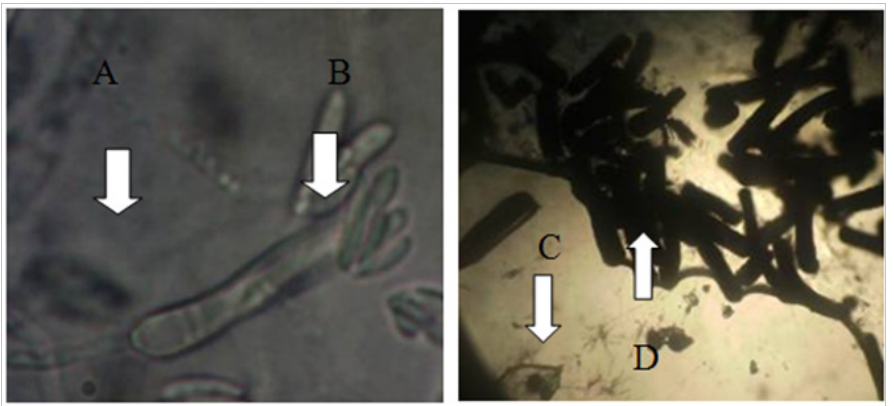

Figure 6 Some features of $C$. lunata under photomicroscope (HPO). Normal hypha (A), conidia (B), broken hyphae (C), broken conidia (D).

Co-culture method: The $C$. cellulolyticum was tested to determine if this fungus could colonize the agar lawn of $C$. lunata using co-culture method as shown in Figure 7. The colonization of C. cellulolyticum was observed on the agar lawn of $C$. lunata by $0.15 \%$ at 30 days as shown in Figure 8, but the pathogen ramified faster at 10 days of incubation. Results revealed that the colonization of $C$. cellulolyticum and C. lunata in co-culture method were $2,867.33 \mathrm{~mm}^{2}$ or $35.40 \%$ and $3,115.33 \mathrm{~mm}^{2}$ or $38.46 \%$ respectively as shown in Figure 9. Based on the results, C. cellulolyticum was grown slowly than the pathogen. In a personal communication with Soytong ${ }^{16}$ at KMITL, Ladkrabang, Thailand, proved that Chaetomium spp. is a slow growing fungus compared to pathogens, and the growth and development is at 25 to 30 days.
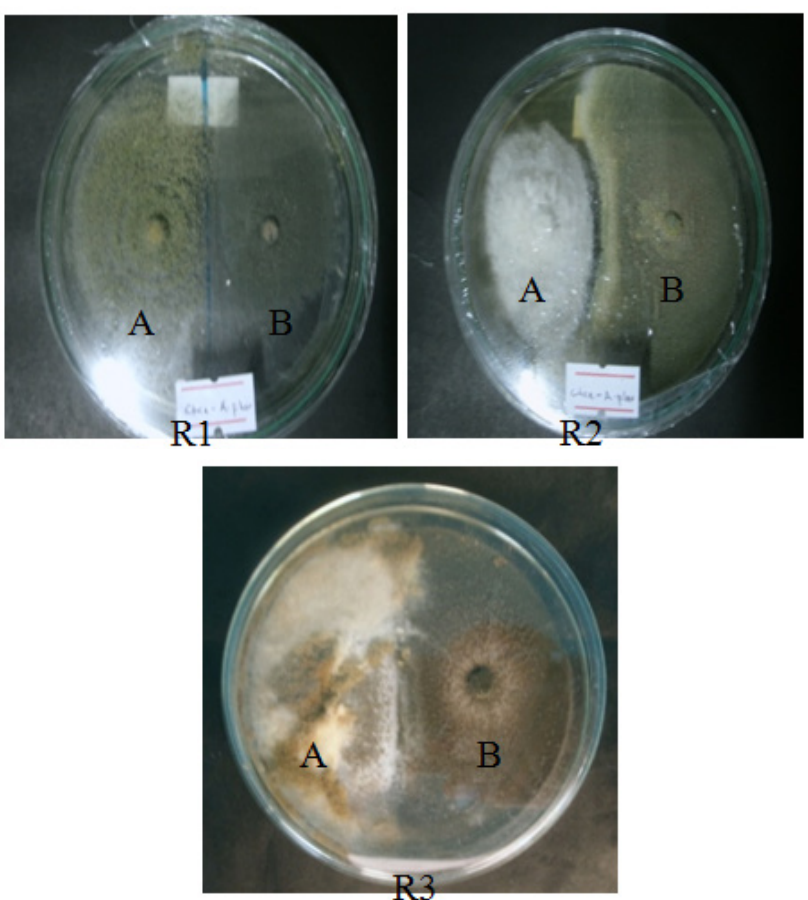

Figure 7 The co-culture of $C$. cellulolyticum (A) and C. lunata (B) per replicates at 30 days. 


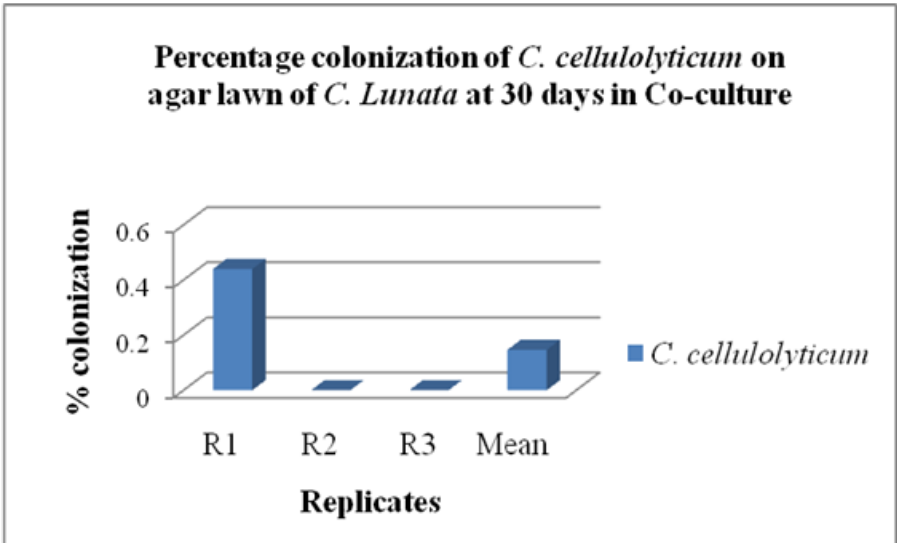

Figure 8 Percentage colonization of $C$. cellulolyticum on agar lawn of C. Lunata at 30 days in Co-culture.

Antimicrobial bioassay: This method was done to determine the inhibitory effect of $C$. cellulolyticum on the mycelial growth of $C$. lunata. Table 2 shows the mean diameter of zone of inhibition of all the treatments. Results revealed that mycelial crude hexane and ethanol extracts do not produced zones of inhibition on C. lunata at $24^{\text {th }}$ to $96^{\text {th }}$ hours of incubation. Analysis of variance and comparison among means showed, that inhibitions of two extracts were comparable to the positive and negative control at $24^{\text {th }}$ hours of incubation. However, at $48^{\text {th }}$ to $96^{\text {th }}$ hours of incubation see (Appendix K-M) the crude extracts were significantly smaller to the positive control but comparable to the negative control. The inhibitory effect of the mycelial crude extracts was not observed at $24^{\text {th }}$ to $96^{\text {th }}$ hours of incubation may because of the small quantity of bioactive compounds in the crude extracts, so the capability to eradicate the pathogen is not possible across time or the pathogen may have already became tolerant or diffused as time of incubation increases. In contrast in study reported by Soytong et al., ${ }^{17}$ that extracts from Chaetomium cupreum and Chaetomium globosum are able to suppress plant pathogens such as Curvularia lunata invitro.

\section{Colony area of C. cellulolyticum and C. lunata at 30} days in Co-culture

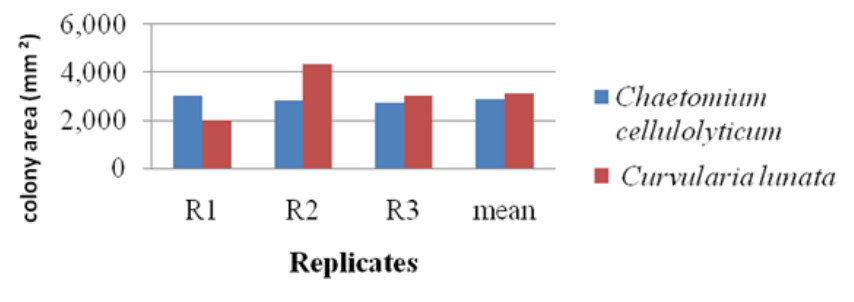

Figure 9 Percentage colonization of $C$. cellulolyticum and C. lunata at 30 days in Co-culture.

Table 2 Diameter of zone of inhibition of all the treatments against Curvularia lunata

\begin{tabular}{|c|c|c|c|c|}
\hline \multicolumn{5}{|c|}{ Mean Diameter of Zone of Inhibition (mm) } \\
\hline Treatments & $24^{\text {th }}$ Hour & $48^{\text {th }}$ Hour & $72^{\text {nd }}$ Hour & $9^{\text {th }}$ Hour \\
\hline TI-Crude Hexane Extract & $6.00^{\mathrm{a}}$ & $6.00^{\mathrm{b}}$ & $6.00^{\mathrm{b}}$ & $6.00^{\mathrm{b}}$ \\
\hline T2-Crude Ethanol Extract & $6.00^{\mathrm{a}}$ & $6.00^{\mathrm{b}}$ & $6.00^{\mathrm{b}}$ & $6.00^{\mathrm{b}}$ \\
\hline T3-Dithane (Positive control) & $6.00^{\mathrm{a}}$ & $42.60^{\mathrm{a}}$ & $41.00^{\mathrm{a}}$ & $35.80^{\mathrm{a}}$ \\
\hline T4-DMSO (Negative control) & $6.00^{\mathrm{a}}$ & $6.00^{\mathrm{b}}$ & $6.00^{\mathrm{b}}$ & $6.00^{\mathrm{b}}$ \\
\hline
\end{tabular}

Means followed by a common letter in each column are not significantly different by DMRT at $\mathrm{P}<0.05$.

\section{Summary}

This study was conducted to test the antagonistic activity of Chaetomium cellulolyticum against Aspergillus flavus and Curvularia lunata using different methods in- vitro. Using Slide bi-culture test, results revealed that the hyphae of A. flavus were deformed and damaged when grows with C. cellulolyticum. Using co-culture method, the results indicated that $A$. flavus ramified faster than $C$. cellulolyticum by $4,234.33 \mathrm{~mm}^{2}$ or $52.28 \%$ at 30 days. In bioassay, results revealed that at $24^{\text {th }}$ to $48^{\text {th }}$ hours of incubation, mycelial crude hexane and ethanol extracts do not produced zone of inhibition on A. flavus. Statistical analysis showed that the zone of inhibition of the two crude extracts were comparable to the positive and negative control. However, at $72^{\text {nd }}$ to $96^{\text {th }}$ hours, the extracts were significantly smaller to the positive control and comparable to the negative control.

On the other hand, the hyphae and conidia of C. lunata were broken as revealed in the Slide bi-culture test. Based on the results of Coculture method, it was observed and measured that the colonization of $C$. cellulolyticum on the agar lawn of $C$. lunata is $0.15 \%$. The colonization of C. cellulolyticum and C. lunata in co-culture method are $2,867.33 \mathrm{~mm}^{2}$ or $35.40 \%$ and $3,115.33 \mathrm{~mm}^{2}$ or $38.46 \%$ respectively. In Bioassay, Results revealed that mycelial crude hexane and ethanol extracts do not produced zones of inhibition on C. lunata at $24^{\text {th }}$ to $96^{\text {th }}$ hours of incubation. Analysis of variance and comparison among means showed, that inhibitions of two extracts were comparable to the positive and negative control at $24^{\text {th }}$ hours of incubation. However, at $48^{\text {th }}$ to $96^{\text {th }}$ hours of incubation, the crude extracts were significantly smaller to the positive control but comparable to the negative control (Figure 10).
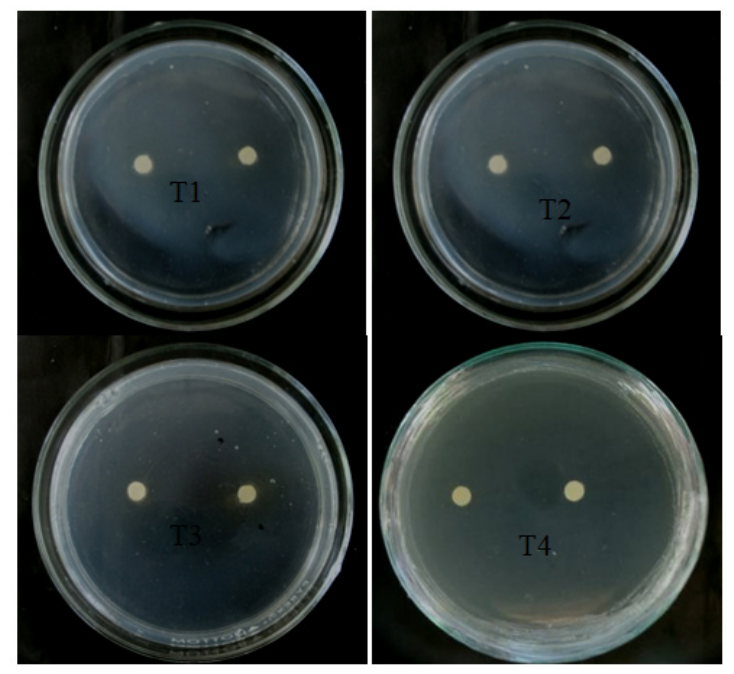

Figure 10 Zone of Inhibition exhibited by all the treatments against $C$. lunato at $24^{\text {th }}$ hours of incubation. (TI- crude hexane extract, T2- crude ethanol extract,T3- positive control (Dithane),T4- negative control (DMSO). 


\section{Conclusion}

Chaetomium cellulolyticum have antibiosis mechanism to deform or damage the morphological hyphae and either conidia of A. flavus and $C$. lunata. However, the affectivity of crude hexane and ethanol extracts from $C$. cellulolyticum were not observed to inhibit the growth of $A$. flavus and C. lunata.

\section{Recommendation}

Chaetomium cellulolyticum have antibiosis mechanism against the pathogens. The product of this study can contribute to conventional and organic farming. However, the following are suggested for further study:

More mycelia of C. cellulolyticum should be extracted and other solvents should be used to test and verify the inhibitory activity. Use other Bioactivity Assay of crude extracts of $C$. cellulolyticum like the method used by Sibounnavong et al. [2]. Measurement of zone of inhibition and determination of spore inhibition should be done in coculture method. Test the different age interval of the C. cellulolyticum and the pathogens in the co-culture method. Use volatile and nonvolatile antibiosis method. Determination of bioactive constituents present in C. cellulolyticum. DNA analysis of C. cellulolyticum should be done. In-vivo biological activities of $C$. cellulolyticum should be done using different tests like, disease suppression, pathogenicity and toxicology tests.

\section{Acknowledgments}

None.

\section{Conflicts of interest}

Authors declare that there is no conflict of interest.

\section{References}

1. Environmental Protection Agency. Pesticides: Regulating Pesticides. Environmental Protection Agency: USA; 2004.

2. Sibounnavong P, Cynthia DC, Kanokmedhakul S, et al. The new antagonist fungus, Emericella nidulans strain EN against Fusarium Wilt of Tomato. Journal of Agricultural Technology. 2008;4(1):89-99.

3. Weber R, Kita W, Runowska HB. Effects of Tillage Methods on The Occurrence of Culm Base Disease In Several Winter Wheat Cultivars. EJPAU. 2001;4(2):06.
4. Ali MK, Abdul ML, Saleem S. Pathogenicity of Lasiodiplodia theobromae and Fusarium solani On Mango. Pak J Bot. 2002;36(1):181-189.

5. Shu-Qin X, Xue C, Ying S. Nature of Physical and Chemical and Pathogenic Function of Toxin of Curvularia lunata(Wakker) Boed. Journal of Maize Sciences; 2006.

6. Alwala S. Identification of Molecular Markers Associated with Resistance to Aspergillus flavus in Maize; 2007.

7. Smith CA, Robertson D, Yates B, et al. The effect of temperature on Natural Antisense Transcript (NAT) expression in Aspergillus flavus Curr Genet. 2008;54(5):241-269.

8. Tsukiboshi A. Diseases of Forage Crops; 2001.

9. Zhang H, Yang Q. Expressed sequence tags-based identification of genes in the biocontrol agent Chaetomium cupreum. Appl Microbiol Biotechnol. 2007;74(3):650-658.

10. Charoenporn C, Kanokmedhakul S, Lin F, et al. Evaluation of bio-agent formulations to control Fusarium wilt of tomato. African Journal of Biotechnology. 2010;9(36):5836-5844.

11. Aggarwal R, Sharma V, Kharbikar L, et al. Molecular characterization of Chaetomium species using URP-PCR. Genetics and Molecular Biology. 2008;31(4):943-946.

12. Soytong K. Antagonism of Chaetomium cupreum to Pyricularia oryzae. Journal of Plant Protection in the Tropics. 1992;9(1):17-23.

13. Kanokmedhakul S, Kanokmedhakul K, Nasomjai P, et al. Antifungal Azaphilones from the fungus Chaetomium cupreum CC3003. J Nat Prod. 2006;69(6):891-895.

14. Pornsuriya C, Lin C, Kanokmedhakul S, et al. Efficacy of antifungal metabolites from some antagonistic fungi against Pythium aphanidermatum. Journal of Agricultural Technology. 2010;6(2):299-308.

15. Soytong K, Srinon W, Rattanacherdchai K, et al. Application of antagonistic fungi to control anthracnose disease of grape. Journal of Agricultural Biotechnology. 2005;1:33-41.

16. Soytong K. Personal communication; 2011.

17. Soytong K, Kanokmedhakul S, Kukongviriyapa V, et al. Application of Chaetomium species (Ketomium ${ }^{\circledR}$ ) as a new broad spectrum biological fungicide for plant disease control: A review article. Fungal Diversity. 2001;7:1-15 